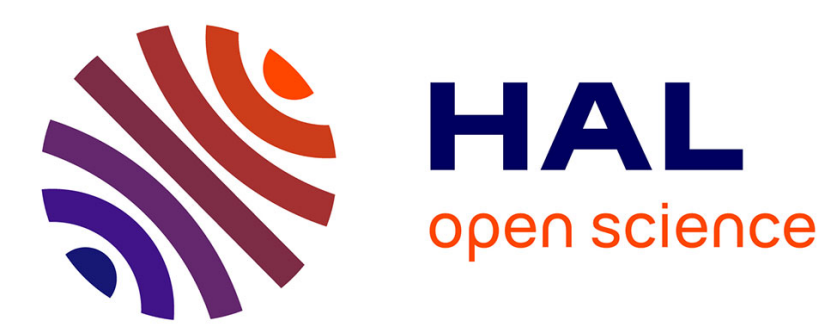

\title{
Robust SM Observer for COVID data prediction and Parameters Estimation
}

Nacer K M’Sirdi, Soufyane Benzaouia, Aziz Naamane

\section{To cite this version:}

Nacer K M'Sirdi, Soufyane Benzaouia, Aziz Naamane. Robust SM Observer for COVID data prediction and Parameters Estimation. ICSC 2021: 9th International Conference on Systems and Control, Jan 2021, Caen, France. hal-03543199

\section{HAL Id: hal-03543199 \\ https://hal.science/hal-03543199}

Submitted on 25 Jan 2022

HAL is a multi-disciplinary open access archive for the deposit and dissemination of scientific research documents, whether they are published or not. The documents may come from teaching and research institutions in France or abroad, or from public or private research centers.
L'archive ouverte pluridisciplinaire HAL, est destinée au dépôt et à la diffusion de documents scientifiques de niveau recherche, publiés ou non, émanant des établissements d'enseignement et de recherche français ou étrangers, des laboratoires publics ou privés. 


\title{
Robust SM Observer for COVID data prediction and Parameters Estimation
}

\author{
Nacer K. M'Sirdi \\ Soufyane Benzaouia \\ SASV / LIS \\ Aix Marseille University \\ Marseille, France
}

Aziz Naamane

\begin{abstract}
The prediction and observation of the growth, and the peaks reproduction of the Coronavirus are of main importance. In this study, we revisit the old deterministic SIR model and show its ability to describe the disease spread; Then we use it to try some observers to avoid acquisition perturbations and measurements imperfections. After finite time converging observations an output re-injection is used for parameters estimation. Note that the data are not provided by sensors which are regular in their measurements, synchronised in time and robust versus noise
\end{abstract}

Index Terms-Compartment models of virus propagation, Observation and prediction, Data filtering, Parameters estimation, Compensation of measurements imperfection and perturbations, Super-Twisting Algorithm (STA).

\section{Introduction}

\subsection{COVID history}

The epidemic spread in a country depends on several parameters. It depends on the population, its density, practices and lifestyles as well as the movements of individuals and the strains of the disease. Understanding the phenomenon of its spread is complex and goes through many stages [1].

Already in 1914, Anderson Gray McKendrick and William Ogilvy Kermack proposed compartmental models, which they revised and extended a few years later [2]-[5]. A large host of mathematical models have followed and continue to evolve until today [6]-[14].

The most common symptoms are fever, dry cough and severe breathing difficulties [1]. The virus is mainly spread step by step during close contact via small droplets resulting from coughing, sneezing or spitting during conversations [15].

People affected by the virus may be asymptomatic or develop flu-like symptoms [1]. Healthy carriers are not easily spotted and remain prone to transmit the virus to others [11]. This is why the fight against COVID-19 is difficult and not yet finished [16]. A new wave of pandemic is problematic, hence the importance of testing.

The goal, in this paper, is to propose efficient observers with reduced complexity and then try to detect and identify the different variables that promote or slow down the spread of the virus [16].

\subsection{Context and formulation}

Measurement imperfections and perturbations can be observed in the available COVID19 data. Data are collected and communicated by human beings doing their best, in addition to their jobs. Then they are gathered, registered and published. To understand the disease evolution in time and in function of some parameters that may seem to be relevant, data processing and observers are needed. Errors and perturbations may occur at each one of theses steps. The Figure 1 shows the daily confirmed cases for three

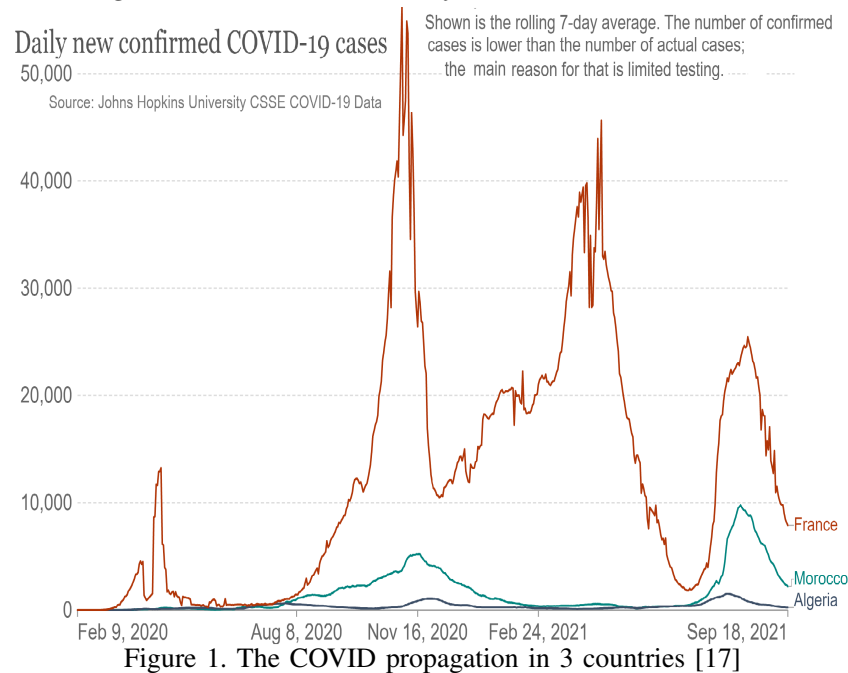

countries. These numbers are reported by institutions and transmitted manually. One can compare some curves. the curves depend on the location and various parameters related to the population and the region particularities. The count of infected people (I) and individuals in remission (R) is not achieved in the same way (the same rigour) in each region or each country. Communication delays and measurement imperfections may exist in the data.

One can note that the numbers (measurements) may have drifts in their dates. This comes from transmissions delays and long reporting chains that exist to gather data statistics. It is worthwhile to note also that the health testing is not 
uniformly and homogeneously done. So errors and time delays exist in the daily numbers [16]. This also means that some values can be overestimated one day and under estimated the next ones. In Figure 2, we remark that there are 30000

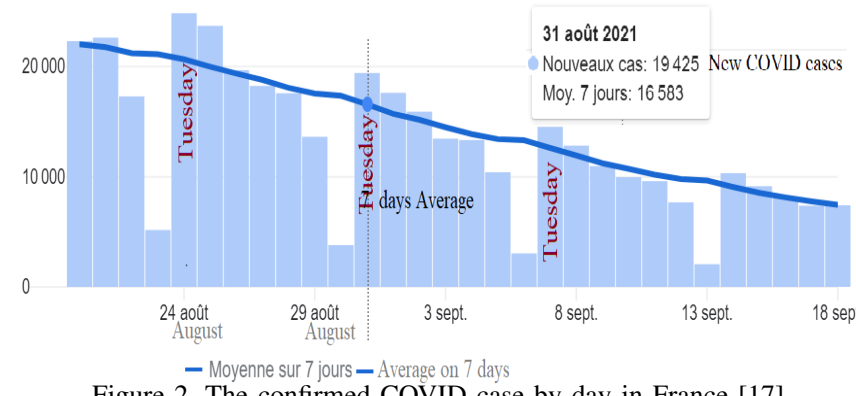

Figure 2. The confirmed COVID case by day in France [17]

periodic perturbations appearing each week for the french data [17].

In this paper we recall the SIR compartment models in section two. Then we try to adapt the model to the actual situation where we observe periodic reproduction of the peak of the infected people number. Section three proposes an appropriate model formulation which allows the design of robust observers by the Sliding Mode (SM) Super Twisting Algorithm (STA). It proceeds by the STA design for the Modified SIR model and then give a discrete time observer in two steps to estimate first the $\mathrm{R}$ and $\mathrm{I}$ numbers and then deduce the $\mathrm{S}$. The 2 most important features of this observation method are the convergence rate of observations and possibility of output injection which allows a final stage of parameters identification. This is used in section four to identify the process parameters. The final thought, as a perspective of this work, is about an approach, or attempt in modelling, for the prediction and the evaluating of some actions that can be taken.

\section{Models in Use}

\subsection{The Compartments Model (SIR)}

2.1.1. SIR Model Definition. The compartment models $S$, I , R, D, E are well presented in Wikipedia. The SIR model is made of at least 3 stages of the disease (S, I, R).

\section{Definition 1.}

- $\mathrm{S}(\mathrm{t})$ is the sub-population susceptible to acquire the disease,

- $\mathrm{I}(\mathrm{t})$ is the sub-population that has become infected,

- $\mathrm{R}(\mathrm{t})$ is the sub-population that has recovered from infection.

Assumption 1. In general, we assume that

- The numbers of Births and Deaths are equal

- The total population is constant: $N=S+I+R=$ cst

- Then the time derivative is null and Births Deaths $=0 . \dot{N}=\dot{S}+\dot{I}+\dot{R}=0$
- Persons who has not catched the COVID is susceptible to be infected (Assumption for SARS-CoV-2)

- The demographic processes variations is neglected (numbers of births and deaths are equal).

- The Recovered sub-population is no longer susceptible to be infected, nor to be infected

- The three sub population groups (S, I and R) are assumed to be non-overlapping.

The epidemic progression have then be modelled by some transition rates as follows

$$
\text { Susceptible } \stackrel{\frac{\beta S I}{N}}{\longrightarrow} \text { Infectious } \stackrel{\gamma I}{\longrightarrow} \text { Recovered }
$$

The infected people I, have $\frac{\beta S I}{N}$ chances to encounter the susceptible population $S$ resulting in infection transmission.

$S$ and $I$ are the sizes of the suspected and infected populations. $\gamma$ is the percentage to recover and become resistant to infection. The Recovered population is $R=\gamma . I$.

For simplicity we use normalised variables or population fractions. Let us define

$$
s=\frac{S}{N} \quad i=\frac{I}{N} \quad r=\frac{R}{N}
$$

The SIR propagation model can be written as follows, where $s+i+r=1$ is an invariant [9], [10].

$$
\frac{d}{d t}\left[\begin{array}{c}
s \\
i \\
r
\end{array}\right]=\left[\begin{array}{ccc}
-\beta . i & 0 & 0 \\
0 & \beta s-\gamma & 0 \\
0 & \gamma & 0
\end{array}\right]\left[\begin{array}{l}
s \\
i \\
r
\end{array}\right]
$$

This model gives us the following curves in simulation, with $R_{0}=2.4, t_{\text {infective }}=8.4, \gamma=1 / t_{\text {infective }}$ and $\beta=R_{0} \gamma$. The initial conditions are: $i_{0}=1 / 200, r_{0}=0.0$ and $s_{0}=$ $1-i_{0}-r_{0}$. (see the reference of prof Jeffrey Kantor [10]

\subsubsection{Conclusion on results of the SIR model. :}

- The constraint $1=\mathrm{r}+\mathrm{s}+\mathrm{i}$, plotted in red is fulfilled

- The infected population shows only one peak and goes to 0

- More than $85 \%$ of the population recovers from infection - The Susceptible population decays to less than $15 \%$

One can note several contradiction with the actual data. The number of infected people decreases to zero after the peak and stays there (indicating that the infection has disappeared). This is not what we observe in reality. The peak came back 3 more times and is coming back again.

At the end of the simulation, there remains a little more than $15 \%$ of the Susceptible population and the rest (ie about $85 \%)$ is the Remitted population. This will mean that the infection will affect a very large majority of the population (here $85 \%$ after only one peak). This also is not the case in the observed actual data.

The population remains constant and the Susceptible number $\mathrm{S}$ reaches up to $85 \%$ of the population. Then, clearly, the dynamics of the COVID19 is not described by this model with the previous assumptions. Only $15 \%$ of the population remain susceptible without evolution of the infection over time! 

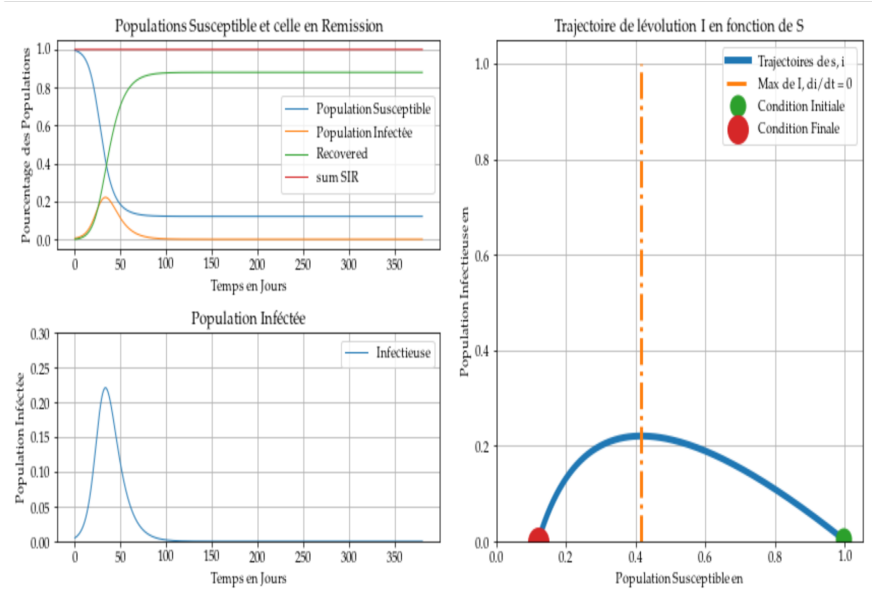

Figure 3. Results with SIR Model by Jeffrey Kantor [10]

More complex models can be considered like the generalised SEIRD model presented in [18], among others but this will not solve the latest problems.

\subsection{The Modified SIRD Model (MSIRD)}

The compartments are more coupled than assumed previously and the epidemic progression have to be modelled by more transition rates.

\subsubsection{MSIRD Model Definition. :}

In this paper we will still consider the simplest SIR model. But we do not keep some of the restrictive assumptions admitted in the previous models. In the proposed model (MSIRD), we assume that there are more transmissions between the compartments.

Assumption 2. For the MSIRD model we assume that:

- We assume that a part of the Recovered subpopulation become Susceptible to the disease and may be (re) infected. We add, to the model, the term $\mu . r$

- The compartments (S, I and R) are coupled. Some infected persons either ignore it or hide it. They are still counted in $S$ instead of in I.

- I is underestimated (leakage -c.i) and $\mathrm{S}$ overestimated (increased by + as).

- The term -d.r is added to account for the small number of deaths in the additional D group.

The proposed modifications lead us to the model equation (MSIRD) (3).

$\left[\begin{array}{c}\frac{d}{d t} s \\ \frac{d}{d t} i \\ \frac{d}{d t} r\end{array}\right]=\left[\begin{array}{ccc}a-\beta . i & 0 & \mu \\ 0 & \beta s-\gamma-c & 0 \\ 0 & \gamma+c & -(\mu+d+a)\end{array}\right]\left[\begin{array}{c}s \\ i \\ r\end{array}\right]$

The MSIRD model (eq (3)) gives us the following curves (Figure 4) in simulation, with the same (previous) parameters and initial states with addition to $m u=0.003, a=0.01$, $c=0.1$ and $\mathrm{d}=0.00001$. The initial number of Infected is $i_{0}=1 / 2000$.

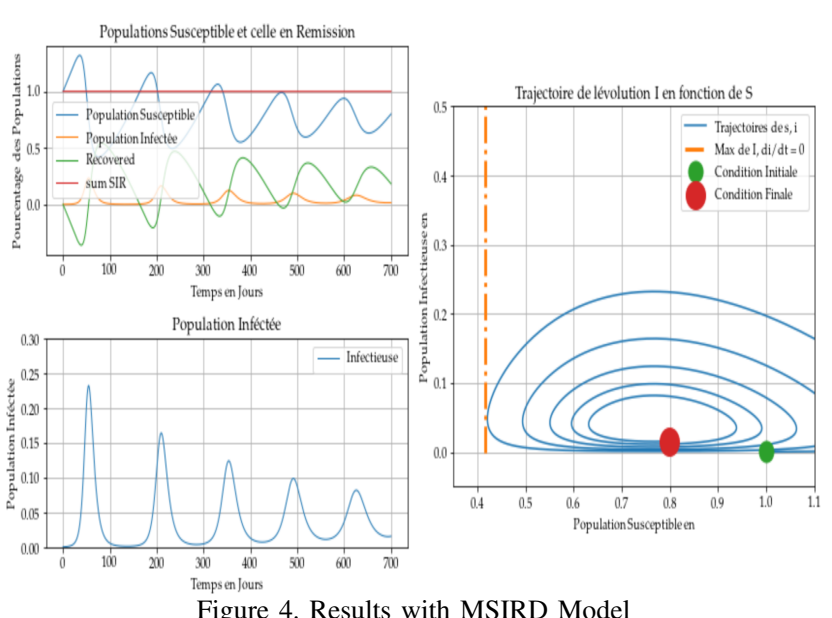

Figure 4. Results with MSIRD Model

2.2.2. Conclusion on results of the MSIRD model. The number of infected people shows periodic peaks with decreasing amplitudes (indicating that the infection comes back quasi-periodically and doesn't disappear) like we observe it reality. The process shows Lotka-Volterra like periodic cycles. The modified MSIRD model is then more suitable to describe the COVID19 spread as shown by Figure (4).

The total population $\mathrm{r}+\mathrm{s}+\mathrm{i}=1-\mathrm{dr}$ is plotted in red (in Figure 4). This suggests to add, as input to $S$, the number of births $b$, to compensate the number of deaths.

The constant population constraint can be exploited to reduce the model complexity. The number of system states can be reduced to only two states, knowing that the third state can be got by $\dot{r}=-\dot{s}-\dot{i}$.

\subsubsection{Proposed reduced 2 states Model MSIRD2. :}

Using the constraint of constant population, we get a reduced order model with only two system states owing to the fact that one state can be deduced from the two others and the constraint. Three second order models are then possible. Let us consider the following one, where we choose $\mathrm{s}$ and $\mathrm{i}$ as states $: \dot{r}=-\dot{s}-\dot{i}$ and $r=1-s-i$

If we choose as outputs $i$ and $r$, we get the system state equations:

$$
\begin{gathered}
{\left[\begin{array}{c}
\frac{d}{d t} s \\
\frac{d}{d t} i
\end{array}\right]=\left[\begin{array}{cc}
a-\mu-\beta i & -\mu \\
0 & \beta s-\gamma-c
\end{array}\right]\left[\begin{array}{l}
s \\
i
\end{array}\right]+\left[\begin{array}{c}
\mu+b \\
0
\end{array}\right]} \\
y=\left[\begin{array}{l}
i \\
r
\end{array}\right]=\left[\begin{array}{cc}
0 & 1 \\
-1 & -1
\end{array}\right] \cdot\left[\begin{array}{l}
s \\
i
\end{array}\right]+\left[\begin{array}{l}
0 \\
1
\end{array}\right]
\end{gathered}
$$

Keeping the same parameters: $\mu=0.003, a=0.01$, $c=0.1$ and $d=0.0001$ with as births number $b=0.00001$, we got the following simulation results. Then no information have been lost by the model reduction. The two state model is enough to describe the complete dynamics as it gives the same results. 


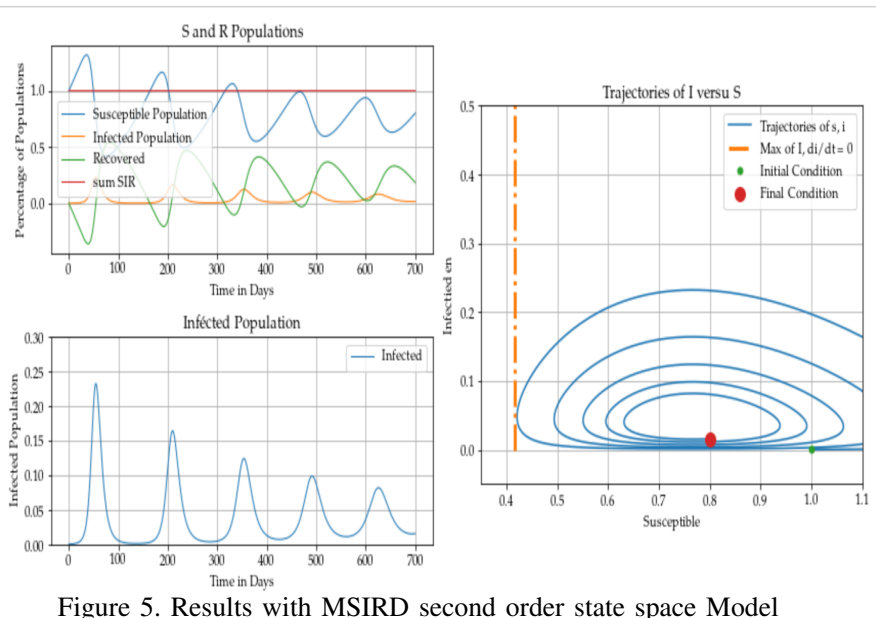

Figure 5. Results with MSIRD second order state space Model

\section{Robust Non Linear State Observer}

\subsection{Parametrization of the process model}

The process variables of interest, which can be quantified, are $\mathrm{i}$ and $\mathrm{r}$. The remaining population $\mathrm{s}$ (hidden state) is not easy to estimate and is of reduced interest to follow the evolution of the infectious disease. It is rather interesting to be controlled. As the global population is constant, s can be deduced from the constraint $s=1-i-r$. We have also $\dot{s}=-\dot{i}-\dot{r}$

Consider the simple nominal model of equation (4). Let us consider the state space vector

$$
X=\left[\begin{array}{l}
x_{1} \\
x_{2} \\
x_{3}
\end{array}\right]=\left[\begin{array}{c}
r \\
\gamma \cdot i \\
s
\end{array}\right]
$$

The variables $x_{1}=r x_{2}=\dot{x}_{1}=\gamma . i$ can be quantified by gathering data application (with $\gamma=1 / t_{\text {infective }}$ [16].

We can write the system model

$$
\begin{aligned}
\dot{x}_{1} & =x_{2} \\
\dot{x}_{2} & =\beta x_{3} x_{2}-\gamma x_{2} \\
\dot{x}_{3} & =-\beta x_{3} x_{2} / \gamma \\
y & =\left[\begin{array}{cc}
x_{1} & 0 \\
0 & x_{3}
\end{array}\right]
\end{aligned}
$$

MSIRD Observer design We have, in two steps, to design an observer for $\left(x_{1}, x_{2}\right)$ and to retrieve $x_{3}$ by using $x_{1}$ and $x_{2}$ with a finite-time (FT) convergence [19]. In a third step, some nonlinear signal are injected for parameters identification (see [20]-[22]).

An output injection signal, filtered from the STA is used to get a regression vector for the RLS (Recursive Least Square) algorithm to identify the parameters asymptotically.

\subsection{Step 1: $x_{1}, x_{2}$ States Estimation}

The subsystem with state variables $x_{1}, x_{2}$, of equation (4) becomes as follows:

$$
\begin{aligned}
& \dot{x}_{1}=x_{2}, \\
& \dot{x}_{2}=f_{1}\left(t, x_{1}, x_{2}\right) \\
& y=x_{1},
\end{aligned}
$$

The dynamics is: $f_{1}\left(t, x_{1}, x_{2}\right)=\beta x_{3} x_{2}-\gamma x_{2}$ is assumed bounded and Lebesgue-measurable.

The proposed observer for system (5) uses the SuperTwisting Algorithm.

Let us design an observer of $x_{2}$ assuming the state $x_{1}=$ $r$ available, which converges in finite-time.

We note $\hat{x}_{1}$ and $\hat{x}_{2}$ the process variables estimations.

The correction variables $z_{1}$ and $z_{2}$ are deduced from the Super-Twisting Algorithm (STA) [23].

The estimation errors are noted $\tilde{x}_{1}=x_{1}-\hat{x}_{1}$ and $\tilde{x}_{2}=$ $x_{2}-\hat{x}_{2}$

The observer can be expressed as in [21]

$$
\begin{aligned}
& \dot{\hat{x}}_{1}=\hat{x}_{2}+z_{1} \\
& \dot{\hat{x}}_{2}=f_{1}\left(t, x_{1}, \hat{x}_{2}\right)+z_{2}
\end{aligned}
$$

The correction terms are computed as

$$
\begin{aligned}
& z_{1}=\lambda\left|x_{1}-\hat{x}_{1}\right|^{1 / 2} \operatorname{sign}\left(x_{1}-\hat{x}_{1}\right) \\
& z_{2}=\alpha \operatorname{sign}\left(x_{1}-\hat{x}_{1}\right) .
\end{aligned}
$$

To ensures observer convergence we take as initial conditions for the estimates $\hat{x}_{1}=x_{1}$ and $\hat{x}_{2}=0$.

The dynamics of the error equations is deduced as

$$
\begin{aligned}
& \dot{\tilde{x}}_{1}=\tilde{x}_{2}-\lambda\left|\tilde{x}_{1}\right|^{1 / 2} \operatorname{sign}\left(\tilde{x}_{1}\right) \\
& \dot{\tilde{x}}_{2}=F\left(t, x_{1}, x_{2}, \hat{x}_{2}\right)-\alpha \operatorname{sign}\left(\tilde{x}_{1}\right)
\end{aligned}
$$

with $F\left(t, x_{1}, x_{2}, \hat{x}_{2}\right)=f_{1}\left(t, x_{1}, x_{2}\right)-f_{1}\left(t, x_{1}, \hat{x}_{2}\right)$

In our process the system states (population fractions) are bounded. Then existence of an upper bound $f^{+}$is ensured such as the inequality (9) holds, for any time and any possible $x_{1}, x_{2}$ and $\left|\hat{x}_{2}\right| \leq 2 \sup \left|x_{2}\right|$.

$$
\left|F\left(t, x_{1}, x_{2}, \hat{x}_{2}\right)\right|<f^{+}
$$

The system states are bounded because the (5) is BIBS stable. $F\left(t, x_{1}, x_{2}, x_{3}, \hat{x}_{2}\right)$ is also uniformly bounded, since all variable $x_{1}, x_{2}, x_{3}$ are uniformly bounded by 1 .

Let us consider $\alpha$ and $\lambda$ two constants verifying the conditions of equations (10), with some constant $p$ is chosen such as $0<p<1$.

$$
\begin{aligned}
& \alpha>f^{+}, \\
& \lambda>\sqrt{\frac{2}{\alpha-f^{+}} \frac{\left(\alpha+f^{+}\right)(1+p)}{(1-p)},}
\end{aligned}
$$

Theorem 1. ( [20], [21]). For the system (5), as the condition

(9) holds, we choose the observer (6) with parameters selected according to conditions (10). Then, the convergence of the estimated states $\left(\hat{x}_{1}, \hat{x}_{2}\right)$ to the real value of the states $\left(x_{1}, \dot{x}_{1}\right)$ is guaranteed after a finite time.

Then there exists a time constant $t_{0}$ such as: for any $t \geq t_{0},\left(\hat{x}_{1}, \hat{x}_{2}\right)=\left(x_{1}, x_{2}\right)$. 
This theorem has been proved by Davila, Fridman and Levant in [20].

The objective now is to get discrete equivalents of the states, the observer and the process variables.

Let us choose as sampling period $\delta$ and let $t_{i}, t_{i+1}$ be two successive times of acquisition.

The variables $x\left(t_{i}\right), z_{1}\left(t_{i}\right), z_{2}\left(t_{i}\right), f_{1}\left(t_{i}\right)$, are discrete times acquisitions with as sampling period $\delta$. Let us consider the Euler discrete equivalent observer

$$
\begin{aligned}
& \hat{x}_{1}\left(t_{i+1}\right)=\hat{x}_{1}\left(t_{i}\right)+\left(\hat{x}_{2}\left(t_{i}\right)+\right. \\
& \left.+\lambda\left|x_{1}\left(t_{i}\right)-\hat{x}_{1}\left(t_{i}\right)\right|^{1 / 2} \operatorname{sign}\left(x_{1}\left(t_{i}\right)-\hat{x}_{1}\left(t_{i}\right)\right)\right) \delta, \\
& \hat{x}_{2}\left(t_{i+1}\right)=\hat{x}_{2}\left(t_{i}\right)+\left(f_{1}\left(t_{i}, x_{1}\left(t_{i}\right), \hat{x}_{2}\left(t_{i}\right)\right)+\right. \\
& \left.+\alpha \operatorname{sign}\left(x_{1}\left(t_{i}\right)-\hat{x}_{1}\left(t_{i}\right)\right)\right) \delta
\end{aligned}
$$

where the estimated variables are $\hat{x}_{1}\left(t_{i}\right)$ and $\hat{x}_{2}\left(t_{i}\right)$

Theorem 2. ( [20]). We assume that $f_{1}$ is a bounded function and that the condition (9) is verified. Then, with the parameters (10), the algorithm (11) ensures the convergent observations with estimation errors such as $\left|\tilde{x}_{1}\right| \leq \gamma_{1} \delta^{2}$, and $\left|\tilde{x}_{2}\right| \leq \gamma_{2} \delta$

where $\gamma_{1}, \gamma_{2}$ are some constants, depending on the observer parameters.

Davila et al have proved also this theorem in [20].

\subsection{Estimation of the State $x_{3}$ (step 2)}

Consider the subsystem with state variable $x_{3}=s$ and

$$
\dot{x}_{3}=-\beta x_{3} x_{2} / \gamma
$$

In this second step, we design an observer, to get a finite-time convergent estimation of the state $x_{3}$ (susceptible population). This leads us an equivalent output injection.

The dynamic equation of $x_{3}$ is written as

$$
\begin{aligned}
& \dot{x}_{3}=f_{2}\left(t, x_{3}\right)+\xi_{2}\left(t, x_{2}, x_{3}\right) \\
& y_{2}=x_{3},
\end{aligned}
$$

In this case, the dynamic of the system considered as unknown is $\xi_{2}\left(t, x_{2}, x_{3}\right)=\beta x_{3} x_{2} / \gamma$, then in consequence $f_{2}\left(t, x_{3}\right)=0$.

The system (12), is assumed bounded and Lebesguemeasurable (Filippov's sense) in any region of the state space.

We note $\tilde{x}_{3}=x_{3}-\hat{x}_{3}$, and recall that $\mathrm{s}$ can be quantified from $s=1-r-i$. Then $x_{3}$ is quantified.

Then we propose the following sliding mode observer

$$
\dot{\hat{x}}_{3}=z_{3}=K \operatorname{sign}\left(x_{3}-\hat{x}_{3}\right)
$$

We can deduce the estimation error dynamic for $x_{3}$ as

$$
\dot{\tilde{x}}_{3}=\xi_{2}\left(t, x_{2}, x_{3}\right)-K \operatorname{sign}\left(\tilde{x}_{3}\right)
$$

with the gain $K$ chosen such as $K>\max \left(\xi_{2}\left(t, x_{2}, x_{3}\right)\right)=$ $\eta$.

Theorem 3. Assume that $\left|\dot{x}_{3}\right| \leq \eta$, and the Sliding Mode gain $K$ is chosen such that $K>\eta$.
Then the observer (13) guarantees, after a finite time, the convergence of the state estimation $\left(\hat{x}_{3}\right)$ to its real value $\left(x_{3}\right)$ transient.

There exists a time constant $t_{1}$ such that for all $t \geq t_{1}$, $\hat{x}_{3}=x_{3}$.

Proof 1. Let us choose as Lyapunov function

$$
V\left(\tilde{x}_{3}\right)=\frac{1}{2} \tilde{x}_{3}^{2}
$$

the time derivative is

$$
\dot{V}\left(\tilde{x}_{3}\right)=\tilde{x}_{3} \dot{\tilde{x}}_{3}=\tilde{x}_{3}\left(\xi_{2}\left(t, x_{2}, x_{3}\right)-K \operatorname{sign}\left(\tilde{x}_{3}\right)\right)
$$

If $K$ is chosen as given in the theorem (3), then the Lyapunov derivative is negative $\dot{V}\left(\tilde{x}_{3}\right)<0$.

This proves that $\tilde{x}_{3}$ goes to zero in a finite time.

Then there exist a constant $t_{1}$ such that for all $t \geq t_{1}$ holds $\tilde{x}_{3}=0$

\section{System Parameters Identification}

\subsection{Equivalent output signal injection}

Recall that the time instants $t_{0}$ and $t_{1}$ are those of Theorems 1 and 3 and let the time $t_{2}=\max \left(t_{0}, t_{1}\right)$.

For all $t \geq t_{2}$ the error dynamics (8) and (14) holds

$$
\begin{gathered}
\dot{\tilde{x}}_{2}=0=F\left(t, x_{1}, x_{2}, \hat{x}_{2}\right)-\alpha \operatorname{sign}\left(\tilde{x}_{1}\right) \\
\dot{\tilde{x}}_{3}=0=\xi_{2}\left(t, x_{2}, x_{3}, u\right)-\beta \operatorname{sign}\left(\tilde{x}_{3}\right)
\end{gathered}
$$

We assume that $z_{2}, z_{3}$ change at a high frequencies. Various imperfections make the state oscillate in some vicinity of the sliding surface intersection. The components of $z_{2}, z_{3}$ are switched at a finite frequency. Then the signals oscillations have high and slow frequency components [24].

The signal $z_{2}, z_{3}$ are (low-pass) filtered to drop their high frequency components. The sliding mode dynamic is determined by the slow components [20]. Then the equivalent control is driven by the slow components. Those are obtained by filtering the high-frequency using a low pass filter (with a sufficiently small time constant). This is to preserve the slow components undistorded enough and to eliminate the high frequency component.

Thus the conditions $\tau \rightarrow 0$ where $\tau$ is the filter time constant, and $\delta / \tau \rightarrow 0$, where $\delta$ is the sample interval, fulfilled to extract the slow component equal to the equivalent control and to filter out the high frequency component.

This allows us to write the equivalent output injection $\bar{z}_{2}$ and $\bar{z}_{3}$ as the filtered versions of $z_{2}$ and $z_{3}$ respectively.

$$
\begin{array}{r}
\bar{z}_{2}=f_{1}\left(t, x_{2}, x_{3}\right)=\beta x_{3} x_{2}-\gamma x_{2} \\
\bar{z}_{3}=\xi_{2}\left(t, x_{2}, x_{3}\right)=-\beta x_{3} x_{2} / \gamma
\end{array}
$$




\subsection{Model for Parameters Identification}

With the parameters $\theta_{1}=[\beta, \gamma]^{T}, \theta_{2}=\beta / \gamma$ and the regression vectors $\varphi_{1}(x)=\left[x_{2} . x_{3},-x_{2}\right]^{T}, \varphi_{2}(x)=-x_{2} x_{3}$ defined below, the process equation (4) is rewritten in an appropriate regression form for parameters identification. We can then use the output injection for the parameters estimation

The Least Squares Estimation (LSE) algorithm [25], [26] can be applied using $\bar{z}_{2}$ and the regression vector deduced from the states observations. For all $t \geq t_{2}$ equations (18), (19) become

$$
\begin{array}{rc}
\bar{z}_{2} & =\hat{\theta}_{1}^{T} \cdot \varphi_{1}\left(\hat{x}_{2}, \hat{x}_{3}\right) \\
\bar{z}_{3} & =\hat{\theta}_{2}^{T} \cdot \varphi_{2}\left(t, \hat{x}_{2}, \hat{x}_{3}\right) \\
\varepsilon_{1}(t)= & \widetilde{\theta}_{1}^{T} \varphi_{1}\left(\hat{x}_{2}, \hat{x}_{3}\right) \\
\varepsilon_{2}(t)= & \widetilde{\theta}_{2}^{T} \varphi_{2}\left(\hat{x}_{2}, \hat{x}_{3}\right)
\end{array}
$$

where $\widehat{\theta}_{i}$ is the estimation of $\theta_{i}$ and $\widetilde{\theta_{i}}$ the estimation error. The LSE estimations, for the two parameters vector, are

$$
\begin{array}{rcc}
\dot{\hat{\theta}}= & \frac{\sigma}{\gamma_{t}} \Gamma_{t} \varphi_{1}\left(\hat{x}_{2}, \hat{x}_{3}\right) \varepsilon(t)=\Gamma_{t} \varphi_{1} \varphi_{1}^{T} \widetilde{\theta_{1}} \\
\dot{\Gamma}_{t}= & -\frac{\sigma}{\gamma_{t}} \Gamma_{t} \varphi_{1}^{T}(t) \varphi_{1}(t) \Gamma_{t} \\
\gamma_{t}= & 1+\varphi_{1}(t) \Gamma_{t} \varphi_{1}^{T}(t)
\end{array}
$$

We use a forgetting factor $\sigma \in[0.9,1]$.

The LSE initial adaptation gain matrix is $\Gamma_{0}=\rho^{-1} I$ and $\widehat{\theta}=\widehat{\Delta \vartheta_{0}}$ are initial parameters values.

Theorem 4. The Recursive Least Squares Estimation algorithm ensures the following properties [25], [27]:

(i) $\widetilde{\theta}^{T} \Gamma_{t}^{-1} \widetilde{\theta}$ is a non increasing and $\left\|\widetilde{\theta}_{t}\right\|^{2} \leq$ $\frac{\lambda \min \left(\Gamma_{O}\right)}{\lambda \max \left(\Gamma_{t}\right)}\left\|\widetilde{\theta}_{0}\right\|^{2}$

(ii) $\tilde{\varepsilon}(t)=\left(\frac{\sigma}{\gamma_{t}}\right)^{1 / 2}(h(t)-\hat{h}(t)) \in L_{2}$

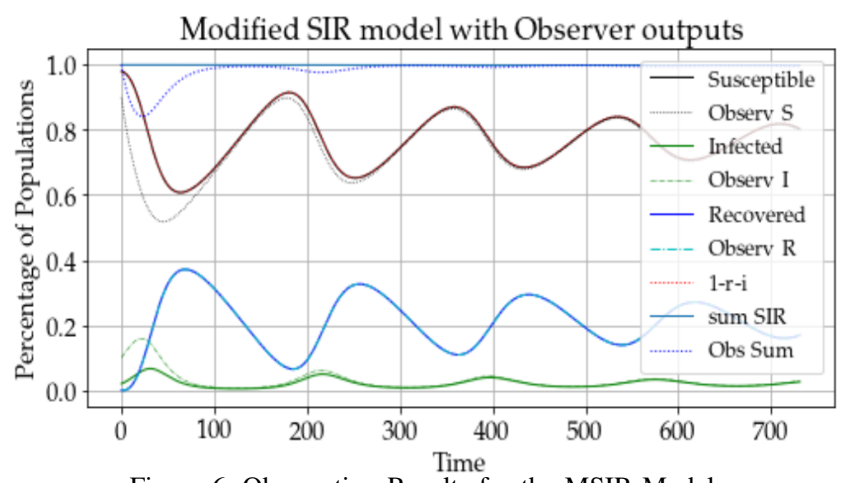

Figure 6. Observation Results for the MSIR Model

Remark 1. The use of LSE ensures the asymptotic convergence of $\widehat{\theta}$ to $\theta$ under the persistent excitation condition.

\section{Simulations and experimental results}

In this section, to validate our approach, some simulation results are presented. The figure (6) shows one of several trials have been done with the proposed MSIRD simulation model and a state estimation observer. It shows the convergence of the estimations.

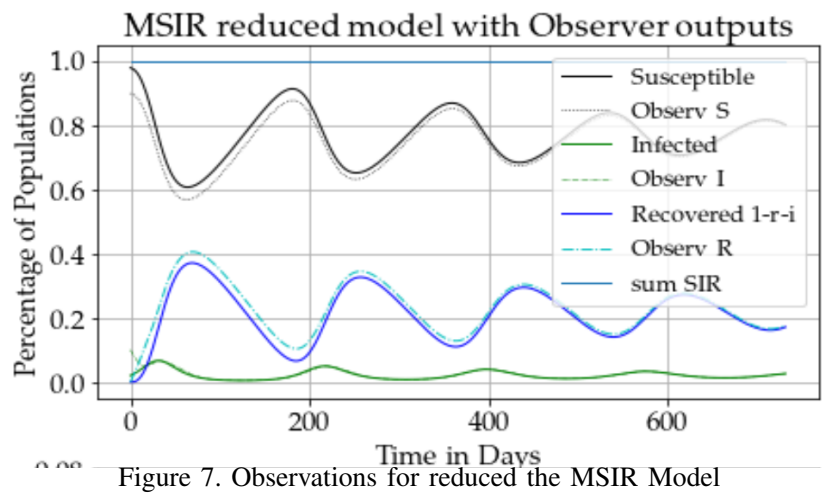

The real data must be pre-processed in order to give interesting and coherent results. The obtained results are very convincing and show that the most efforts have to be done for the data prediction. We have used median filtering to remove the pics and outliers. An averaging filter, with a 7 days sliding window, is used to remove the one week periodicity (see fig 1) due to data acquisition features. The given daily new-cases are compared to derivatives of totalcases (for I, R Data) and smoothed to fit the progression curves.

\section{Conclusion}

The $2^{d}$-order sliding-mode (super-twisting algorithm) is used to define (in two steps) a states estimation for COVID propagation model and data. The convergence of the estimations, in finite time, permits the step by step state estimation and to avoid lack of sensors to estimate s. Then parameters identification is made possible in a third step. This observer is shown to be robust. Its robustness allows us to reconstruct non-measurable variables. Cascaded robust estimators converging in finite time to help to avoid absence of sensors.

After robust state estimations converging in finite time, we can retrieve the rejected signals which can be used as an output injection for some parameters estimation (thanks to the rejection property and robustness). Least Squares Estimation (LSE) method can then be used for the process parameters estimation.

The predictive observations and estimations will be mixed, in perspectives, to online hypothesis testings to reduce the data pre-processing burden and forward predictions.

\section{Acknowledgment}

Thanks to the SASV research group of the LIS laboratory for authorizing this free and unsupported research 


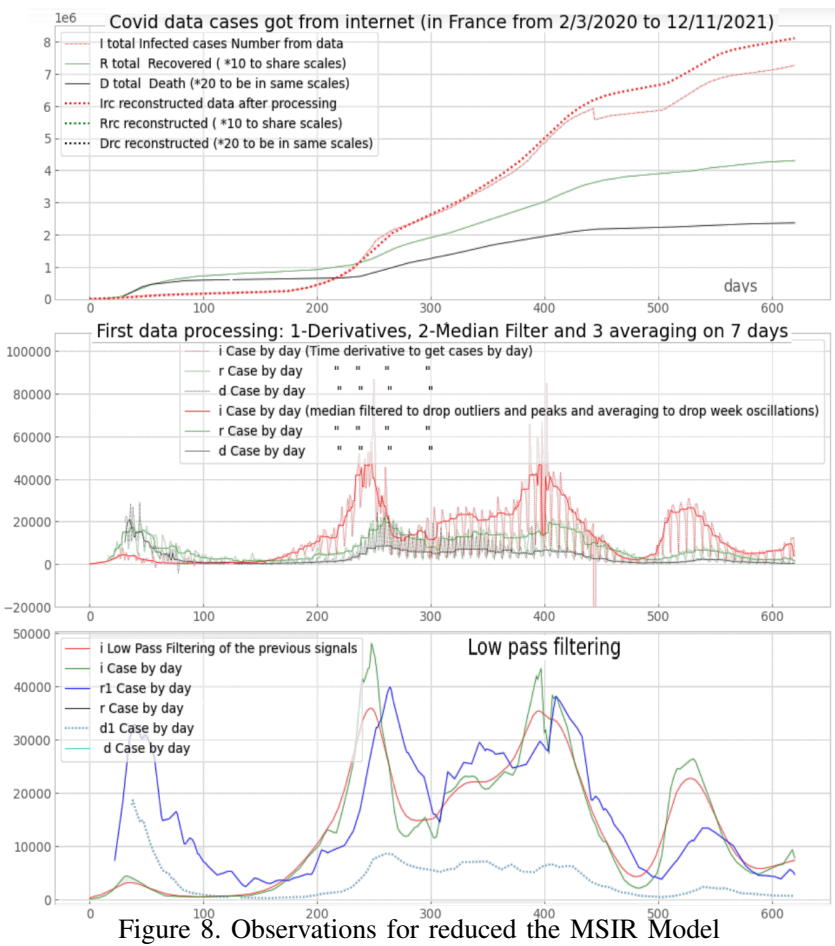

activity. This work is dedicated to memory of our friend Abd El Kebir Boukas. Thanks to the reviewers for their good comments.

\section{References}

[1] P. Wang and J. Jia, "Stationary distribution of a stochastic sird epidemic model of ebola with double saturated incidence rates and vaccination." Advances in Difference Equations, no. 433, pp. 1-16, 2019. [Online]. Available: https://doi.org/10.1186/ s13662-019-2352-5

[2] A. G. Mc Kendrick, "Studies on the theory of continuous probabilities with special reference to its bearing on natural phenomena of a progressive nature," Proceedings of the London Mathematical Society, vol. s2-13, no. 1, pp. 401-416, 1914. [Online]. Available: https://londmathsoc.onlinelibrary.wiley.com/doi/ abs/10.1112/plms/s2-13.1.401

[3] _ - "Applications of mathematics to medical problems," Proceedings of the Edinburgh Mathematical Society, vol. 44, p. 98-130, 1925.

[4] W. Kermack and A. M. Kendrick, "A contribution to the mathematical theory of epidemics," Proc. R. Soc. Lond. B Biol. Sci., vol. 115, p. 700-721, 1927.

[5] W. Kermack and A. Mc Kendrick, "Contributions to the mathematical theory of epidemics, part. iii," Proc. R. Soc. Lond. B Biol. Sci., vol. 141, p. 94-112, 1932.

[6] D. Aleman, T. Wibisono, and B. Schwartz, "Accounting for individual behaviors in a pandemic disease spread model," in Proceedings of the 2009 Winter Simulation Conference (WSC), 01 2010, pp. 1977 - 1985.

[7] C. Abbott, E. Haseltine, R. Martin, and J. Hedengren, "New capabilities for large-scale models in computational biology," Computing and Systems Technology Division, AIChE National Meeting, 2012.

[8] T. Berge, J.-S. Lubuma, G. Moremedi, N. Morris, and R. KonderaShava, "A simple mathematical model for ebola in africa," Journal of Biological Dynamics, vol. 11, no. 1, pp. 42-74, 2017, pMID: 29067875. [Online]. Available: https://doi.org/10.1080/17513758. 2016.1229817
[9] A. Džiugys, M. Bieliunas, G. Skarbalius, E. Misiulis, and R. Navakas, "Simplified model of covid-19 epidemic prognosis under quarantine and estimation of quarantine effectiveness," medRxiv, 2020. [Online]. Available: https://www.medrxiv.org/content/early/2020/06/ 17/2020.04.28.20083428

[10] J. Kantor, "Modeling and control of a campus covid-19 outbreak," https://jckantor.github.io/covid-19/, 2020, https://youtu.be/057Ev6cKLwE?t=1930. [Online]. Available: https: //github.com/jckantor/covid-19

[11] C. Gourieroux and J. Jasiak, "Time varying markov process with partially observed aggregate data; an application to coronavirus," Working Papers 2020-11, Center for Research in Economics and Statistics, 2020, revised 08 May 2020.

[12] R. M. Ziff and A. L. Ziff, "Fractal kinetics of covid-19 pandemic." medRxiv (2020), 2020.

[13] A. Oustaloup, F. Levron, S. Victor, and L. Dugard, "Non-integer (or fractional) power model of a viral spreading: application to the covid$19, " 2021$.

[14] J. Rémond and Y. Rémond, "Sur une modélisation simplifiée de l'épidémie du Covid-19 de 2020," Apr. 2020, working paper or preprint. [Online]. Available: https://hal.archives-ouvertes. fr/hal-02551464

[15] R. Yaesoubi and T. Cohen, "Generalized markov models of infectious disease spread: A novel framework for developing dynamic health policies," European journal of operational research, vol. 3, no. 215, pp. 679-687, 2011, pMID: 21966083; PMCID: PMC3182455.

[16] J. H. University, "The covid-19 data repository by the center for systems science and engineering (csse) at johns hopkins university (jhu)," 2021. [Online]. Available: https://github.com/owid/ covid-19-data/tree/master/public/data

[17] M. Roser, H. Ritchie, E. Ortiz-Ospina, and J. Hasell, "Coronavirus pandemic (covid-19)," Our World in Data, 2020. [Online]. Available: https://ourworldindata.org/coronavirus

[18] L. Peng, W. Yang, D. Zhang, C. Zhug, and L. Hong, "Epidemic analysis of covid-19 in china by dynamical modeling," in arXiv preprint arXiv:2002.06563, 2020. [Online]. Available: https: //www.medrxiv.org/content/10.1101/2020.02.16.20023820v1

[19] A. Polyakov and L. Fridman, "Stability notions and lyapunov functions for sliding mode control systems," Journal of the Franklin Institute, vol. 351(4), p. 1831-1865, 2014.

[20] J. Davila, L. Fridman, and A. Poznyak, "Observation and identification of mechanical systems via second order sliding modes," International Journal of Control, vol. 79(10), p. 1251-1262, 2006.

[21] N. M'sirdi, A. Rabhi, L. Fridman, J. Davila, and Y. Delanne, "Second order sliding mode observer for estimation of velocities, wheel sleep, radius and stiffness," in In Proceedings of the 2006 American Control Conference, Minneapolis, Minnesota USA, 2006, p. 3316-3321.

[22] R. Ushirobira, W. Perruquetti, M. Mboup, and M. Fliess, "Algebraic parameter estimation of a biased sinusoidal waveform signal from noisy data," IFAC Proceedings Volumes, vol. 45, no. 16, pp. 167-172, 2012, 16th IFAC Symposium on System Identification. [Online]. Available: https://www.sciencedirect.com/science/article/ pii/S1474667015379465

[23] A. Levant, "Robust exact differentiation via sliding mode technique," Automatica, vol. 34(3), pp. 379-384, 1998.

[24] V. Utkin, J. Guldner, and J. Shi, Sliding Mode Control in Electromechanical Systems. London, UK:Taylor \& Francis, 1999.

[25] L. Ljung and J. Soderstrom, System Identification. MIT press, Cambridge, 1983.

[26] C. Goodwin and K. Sin, Adaptive Filtering Prediction and Control. Prentice Hall. New Jersey, 1984.

[27] T. Soderstrom and P. Stoica, System Identification. Cambridge, Great Britain:Prentice Hall International, 1989. 